\title{
Seven-year tolerability profile of glucocorticoids use in early rheumatoid arthritis: data from the ESPOIR cohort
}

\author{
Camille Roubille, ${ }^{1,2}$ Nathalie Rincheval, ${ }^{1,3}$ Maxime Dougados, ${ }^{4,5}$ René-Marc Flipo, ${ }^{6}$ \\ Jean-Pierre Daurès, ${ }^{3}$ Bernard Combe ${ }^{1}$
}

Handling editor Tore K Kvien

- Additional material is published online only. To view please visit the journal online (http://dx.doi.org/10.1136/ annrheumdis-2016-210135)

'Rheumatology Department, Lapeyronie Hospital, Montpellier University, Montpellier, France ${ }^{2}$ Internal Medicine and Hypertension Department, Lapeyronie Hospital, Montpellier University, Montpellier, France ${ }^{3}$ Statistiques, University Institute of Clinical Research, EA2415, Montpellier, France

${ }^{4}$ Paris Descartes University, Rheumatology Department, Cochin Hospital, AP-HP, Paris, France

${ }^{5}$ INSERM (U1153): Clinical Epidemiology and Biostatistics, PRES Sorbonne Paris-Cité, Paris, France

${ }^{6}$ Department of Rheumatology, Roger Salengro Hospital, Lille, France

Correspondence to Professor Bernard Combe, Département de Rhumatologie, Hôpital Lapeyronie, Montpellier cedex 34295, France; b-combe@chu-montpellier.fr

Received 28 June 2016 Revised 14 January 2017 Accepted 21 January 2017 Published Online First 17 February 2017

\section{Linked}

- http://dx.doi.org/10.1136/ annrheumdis-2017-211187

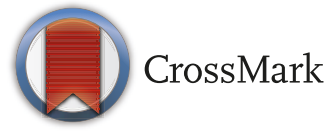

To cite: Roubille C, Rincheval N, Dougados M, et al. Ann Rheum Dis 2017:76:1797-1802.

\section{ABSTRACT}

Objective To explore the 7-year tolerability profile of glucocorticoids (GC) for early rheumatoid arthritis (RA). Methods We examined data for 602 patients with RA from the early arthritis Etude et Suivi des POlyarthrites Indifférenciées Récentes (ESPOIR) cohort ( $<6$ months disease duration) stratified into two groups: with or without GC treatment at least once during follow-up (median 7 years (IQR 0.038-7.65)). The main outcome was a composite of death, cardiovascular disease (including myocardial ischaemia, cerebrovascular accident and heart failure), severe infection and fracture.

Results Among the 602 patients with RA (476 women $(79 \%)$, mean age $48 \pm 12$ years), 386 with GC $(64.1 \%)$ received low-dose prednisone (mean $3.1 \pm 2.9 \mathrm{mg} /$ day for the entire follow-up): 263 started GC during the first 6 months (68\%), and the mean duration of total GC treatment was $1057 \pm 876$ days. As compared with patients without GC (216 (35.9\%)), those with GC showed greater use of non-steroidal anti-inflammatory drugs, synthetic and biological disease-modifying antirheumatic drugs and had more active disease disability, higher $\mathrm{C}$ reactive protein and anticitrullinated protein antibody levels. Among 65 events (7 deaths, 14 cardiovascular diseases, 19 severe infections and 25 fractures), 44 and 21 occurred in patients with and without $\mathrm{GC}(p=0.520)$. Infections were more frequent, although not significantly, in patients with than without GC $(p=0.09)$. On weighted Cox proportional-hazards analysis, with use of propensity score and inverseprobability-of-treatment weighting, and including age, gender, history of hypertension and GC treatment, outcomes did not differ with and without $\mathrm{GC}(p=0.520$; $\mathrm{HR}=0.889 ; 95 \% \mathrm{Cl} 0.620$ to 1.273 ).

Conclusions This 7-year analysis of the ESPOIR cohort supports the good safety profile of very low-dose GC for early active RA.

\section{INTRODUCTION}

Glucocorticoids (GC) are commonly prescribed for patients with rheumatoid arthritis (RA), especially in the early stages of the disease. However, beyond their symptomatic and structural benefits, ${ }^{1}$ the risk/ benefit ratio of GC remains controversial, ${ }^{2}$ with concerns about the tolerability profile. GC adverse events have been reported to be time-dependent and dose-dependent, so international guidelines support the use of the lowest dose for the shortest duration. ${ }^{3} 4$
GC use in RA remains controversial, mostly because of concerns about long-term safety outcomes, including cardiovascular disease (CVD), infection, diabetes, weight gain, osteoporosis and fracture. ${ }^{5}$ Nevertheless, despite the fear of adverse events, GC are widely used in RA, especially in early active diseases, with variable dosage and duration. For instance, in the UK, $50 \%$ of patients with incident RA were reported to receive GC in primary care, with more than $50 \%$ receiving doses $>10 \mathrm{mg} /$ day. $^{6}$ In the German Course And Prognosis of Early Arthritis (CAPEA) inception cohort, $77 \%$ of patients initially received oral GC, $20 \%$ receiving low-dose GC $(<7.5 \mathrm{mg} /$ day $)$ and $35 \%$ high-dose GC ( $\geq 20 \mathrm{mg} /$ day). ${ }^{7}$ By contrast, in the Canadian CATCH cohort, only $42 \%$ of patients started on GC; 48\% received oral GC $(\leq 10 \mathrm{mg} /$ day $)$ and $38 \%$ intra-articular or intramuscular GC. ${ }^{8}$ In another inception cohort from Latin America, $64 \%$ of early patients with RA took GC $\left(80 \% \leq 10 \mathrm{mg} /\right.$ day of prednisone). ${ }^{9}$ Notably, despite the longstanding use of GC for daily RA management, few strong evidence-based safety data are available. ${ }^{10}{ }^{11}$ Therefore, the safety of GC remains on the research agenda, especially the tolerability profile in early RA.

In the Etude et Suivi des POlyarthrites Indifférenciées Récentes (ESPOIR) cohort (see online supplementary method 1$),{ }^{12}{ }^{13}$ more than half of patients received GC at least once over 5 years after inclusion, especially during the first 6 months of follow-up. ${ }^{14}$ Thus, investigation of consecutive events occurring after GC initiation in this cohort may give insight into the long-term safety profile of GC use in a real-life setting.

Here, we aimed to explore the 7-year tolerability profile of GC for patients with recent-onset RA (ESPOIR cohort) by determining the association between GC use and major safety events, including death, CVD, severe infection and fracture.

\section{PATIENTS AND METHODS}

\section{Study design and setting}

The ESPOIR cohort is a French prospective multicentre observational cohort sponsored by the French Society of Rheumatology that included patients (aged 18-70 years) with early arthritis from 14 rheumatology centres in France. To be included, patients had to have inflammatory arthritis in at least two swollen joints lasting from 6 weeks to 6 months, with the potential to develop 
into RA, and be naïve to disease-modifying antirheumatic drugs (DMARDs) and GC therapy. The exclusion criterion was early inflammatory joint disease meeting criteria for a definite diagnosis other than RA or exhibiting features that ruled out progression to RA. ${ }^{12}$ The ESPOIR cohort included 813 patients between 2002 and 2005. The objective, design and characteristics of the cohort were previously described ${ }^{13}$ (see online supplementary method 1). The database for the present study was locked in 2013 at the 7-year time point. The protocol of the ESPOIR cohort was approved by the ethics committee of Montpellier, France (no. 020307). All patients gave their signed informed consent before inclusion.

\section{Patients and GC use}

Among the 813 patients included, we selected the 712 who fulfilled the 2010 American College of Rheumatology/European League Against Rheumatism criteria for $\mathrm{RA}^{15}$ over the 7 years of follow-up (figure 1). We excluded patients with a history of CVD (including myocardial ischaemia, cerebrovascular accident and heart failure), severe infection or fracture because we anticipated that such patients might have had a different profile for GC prescription (expected to less frequently receive a prescription from rheumatologists) and risk of related side effects (likely greater).

We also excluded patients with missing data for GC treatment and those not followed-up to 1 year (figure 1), to provide sufficient monitoring data and a sufficient duration of follow-up to ensure that the impact of GC was a true effect and not due to chance in a short observation period (see online supplementary method 2).

Hence, we examined data for 602 patients with an RA diagnosis. To consider the group of patients with a history of CVD, severe infections and fractures, we also analysed data for 657 patients comprising the 602 included patients and the 55 patients with such a history, and no missing data (figure 1).

The included patients were then classified into two groups by whether or not they received GC at least once (with or without GC) over the 7 years of follow-up. The group with GC comprised patients who received systemic GC treatment (oral, intramuscular or intravenous) at least once between inclusion and the end of follow-up. The group without GC comprised patients who never took GC between inclusion and the end of follow-up. Patients who received only intra-articular injections of GC or inhaled GC were included in the group without GC, ${ }^{16}$ given the minimal systemic spreading.

GC use was defined as use (yes or no) when the first safety event occurred. Doses were calculated as prednisone-equivalent based on accepted standards. ${ }^{17}$ For each patient with GC treatment, the mean dosage of GC was calculated by dividing the total quantity of GC by the duration of the entire follow-up.

\section{Outcomes and variables}

The primary outcome was a composite of new-onset safety events including all-cause mortality, CVD (myocardial
Figure 1 Study design. CVD, cardiovascular disease; ESPOIR, Etude et Suivi des POlyarthrites Indifférenciées Récentes; GC, glucocorticoids; RA, rheumatoid arthritis.

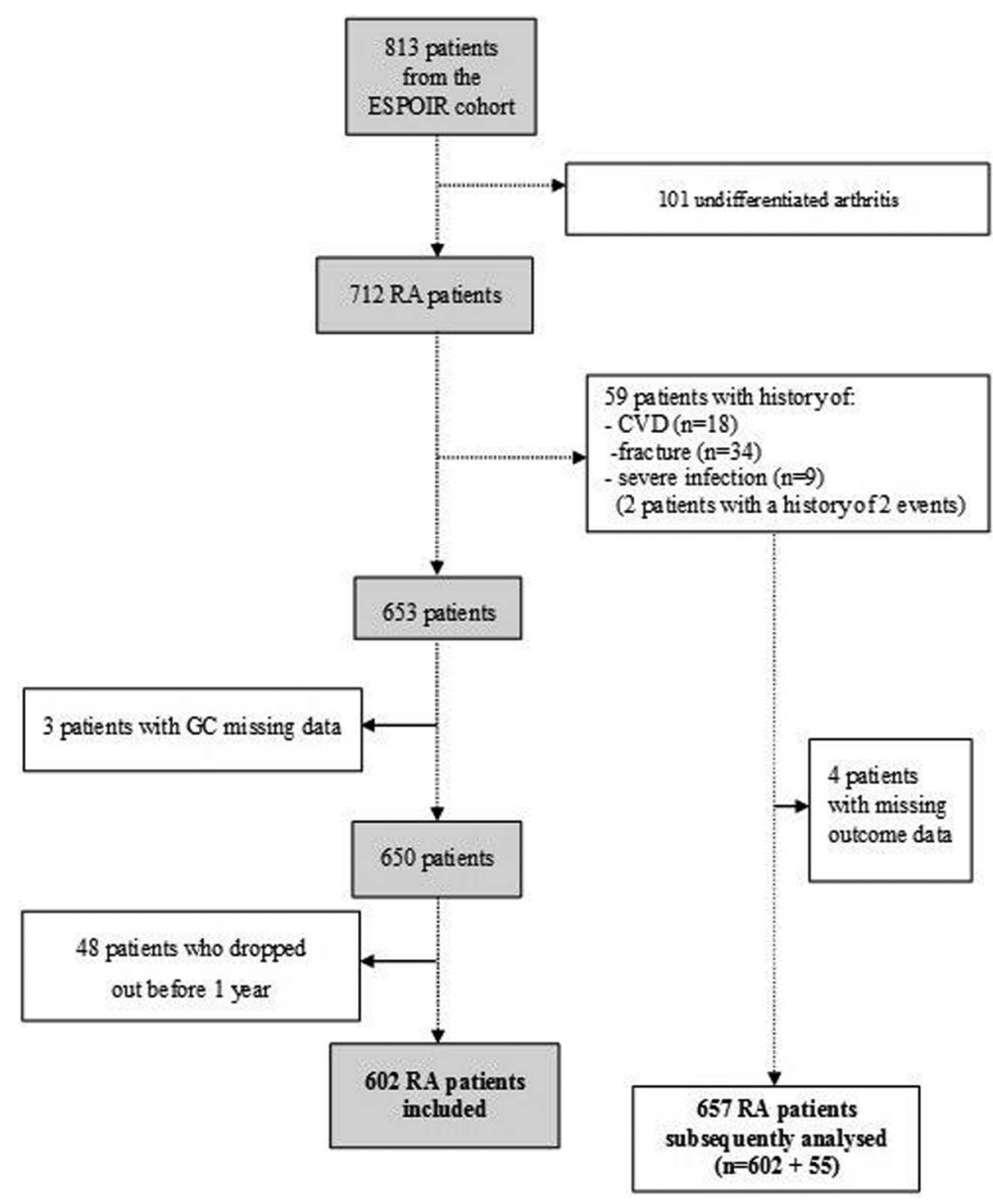


ischaemia, cerebrovascular accident and heart failure), severe infection and fracture. Only new-onset events reported were considered so as to ensure the relevance of the association with GC treatment. We selected as covariates some factors known to affect cardiovascular, infection and fracture risks: age, gender, body mass index (BMI), diabetes, hypertension, smoking status, hypercholesterolaemia and use of non-steroidal antiinflammatory drugs (NSAIDs). A variable named 'cardiovascular risk' was created to account for the presence of at least one cardiovascular risk factor among hypertension, hypercholesterolaemia, BMI $>30 \mathrm{~kg} / \mathrm{m}^{2}$, diabetes and smoking. All all-cause deaths were considered. CVD included myocardial infarction, acute coronary syndrome, angina pectoris, stroke (ischaemic or haemorrhagic) and heart failure. Severe infection was defined as requiring hospitalisation or intravenous antibiotics. Outcomes were recorded in the cohort file.

\section{Statistical analyses}

Descriptive statistics are presented as mean \pm SD or number (\%) where appropriate. The non-parametric Mann-Whitney U test was used to compare the distribution of continuous variables and $\chi^{2}$ test (or Fisher's exact test) to test the association of categorical variables. Continuous variables were transformed into categorical variables with the median or a predetermined threshold. The $\mathrm{p}$ values $<0.05$ were considered significant and all statistical tests were two-sided. The composite primary outcome was compared by $\chi^{2}$ test (or Fisher's exact test) on univariate analysis. Thereafter, Cox proportional-hazards regression was used to assess the association between GC treatment and outcome, estimating HRs and 95\% CIs. To reduce the impact of treatment selection bias and potential confounding, the weighted Cox proportional-hazards model was used with inverse-probability of treatment weighting (IPTW). ${ }^{18}$ With this method, weights for patients who had and patients who had not received GC treatment were the inverse of 'propensity score' (PS) and the inverse of '1-PS', respectively. To account for potential confounding by indication, where patients with more severe disease would be more likely to receive GC, we used a PS. The PS is defined as the predicted patient's probability of receiving GC, conditional on a set of observed baseline covariates. The PS was estimated by multiple logistic-regression analysis. Two sets of observed baseline covariates were included in the PS model (see online supplementary figures S1 and S2). ${ }^{19}$ The first set of covariates, selected by using the log-rank test, was related to the outcome and the second set, selected using $\chi^{2}$ test, was related to GC treatment (see online supplementary figures S1 and S2). Of note, all Disease Activity Score in 28 joints (DAS-28) C reactive protein (CRP) levels were not significant, therefore we preferred including each component of the DAS-28 CRP score separately. For both long-rank and $\chi^{2}$ tests, the level of significance was set at $\mathrm{p}<0.15$. In addition, we used three procedures for selecting variables (forward, backward and stepwise) to obtain the most appropriate logistic-regression equation. All procedures led to the same model. The baseline covariates retained in the final PS regression model were: anticitrullinated protein antibodies (ACPA), diabetes, Health Assessment Questionnaire (HAQ) score, van der Heijdemodified Sharp score (mSHS), cardiovascular risk and patient's overall assessment using visual analogue scale (see online supplementary figure S1). The PS was then included in the Cox proportional-hazards model with the baseline covariates that were significant at $15 \%$ on the log-rank test and not already included in the PS model: age, gender, history of hypertension, in addition to GC treatment (yes or no).
In the analysis of the 657 patients, comprising the 602 patients without a history and the 55 patients with a history and sufficient data, the methodology was similar. The two sets of covariates included in the PS model were the same as in the principal analysis, with, in addition, the covariate history of CVD, severe infection or fracture. The baseline covariates retained in the PS regression model for this analysis were the same, except for mSHS (see online supplementary figure S2). The PS was then included in the Cox proportional-hazards model with age, gender, history of hypertension, BMI $>30 \mathrm{~kg} / \mathrm{m}^{2}$, CRP, rheumatoid factor (RF), history of CVD, severe infection or fracture, in addition to GC treatment (yes or no). Furthermore, the original studied population could be considered different subgroups on the basis of the cumulative GC dose and total duration of GC treatment over 7 years. These two variables were transformed into a four-level categorical variable by quartiles. First, the log-rank test was used to evaluate the effect of the two covariates with time to event data. Moreover, to adjust for such subgroup differences, an extension of the standard Cox model was used to create two stratified Cox models based on the categorical variable levels. $p$ Value $<0.05$ was considered to be statistically significant and all statistical tests were two-sided. All statistical analyses involved use of SAS V.9.3 (SAS Institute, Cary, North Carolina, USA).

\section{RESULTS}

The study population comprised 602 patients with RA (476 women $(79 \%)$, mean age $48 \pm 12$ years; table 1$)$. Mean duration of follow-up was $5.98 \pm 1.84$ years (median 7 years IQR $(0.038$ 7.65)). Baseline characteristics of the entire sample are shown in table 1: $91 \%$ had moderate to high disease activity, and almost $45 \%$ were ACPA-positive. A total of $386(64.1 \%)$ received GC during follow-up and 216 never received GC (35.9\%). Patients with GC mainly received low-dose prednisone during follow-up (mean $3.1 \pm 2.9 \mathrm{mg} /$ day, median $2.4 \mathrm{mg} /$ day (IQR $0.7-5$ ) for the entire follow-up); over half started GC during the first 6 months $(\mathrm{n}=263,68.1 \%)$. Among the 386 patients who received GC, 73 (19\%) received GC for $\leq 6$ months, and 185 (almost 50\%) for 2 years (see online supplementary table S1); 69 patients received GC for up to 6 years (18\%). The mean duration of total GC treatment was $1057 \pm 876$ days (median 803 days (IQR 267$1829)$ ) and $280(72.5 \%)$ received GC continuously for longer than 6 months. Six patients received only intravenous or intramuscular GC. Active disease was greater with than without GC, with significantly higher DAS-28-CRP level and HAQ score, as reflected by a greater consumption of DMARDs, biological agents and NSAIDs and higher CRP levels and ACPA titres (table 1).

\section{Effect of GC exposure: composite of death, CVD, severe infection and fracture}

A total of 65 events were reported in the entire population: 7 deaths, 14 CVD, 19 severe infections and 25 fractures (table 2).

Deaths occurred between years 4 and 7 and were caused by cancer or malignant blood diseases $(n=4)$, salmonellosis $(n=1)$, ruptured aortic aneurysm $(n=1)$ or unknown cause $(n=1)$.

Among these 65 events, 44 (11.4\%) and 21 (9.7\%) occurred in patients with and without GC $(\mathrm{p}=0.520)$ (table 2). The number of infections was greater, although not significantly, in patients with than without GC $(\mathrm{p}=0.09)$.

When we considered patients with events $(n=65)$ and compared patients with and without GC by DAS-28-CRP, those with GC and moderate to high disease activity (DAS-28-CRP score 
Table 1 Baseline demographic, clinical, biological and radiographic characteristics of the study population from the ESPOIR cohort ( $\mathrm{n}=602$ ) and those without and with GC

\begin{tabular}{|c|c|c|c|c|c|}
\hline & $N$ & $\begin{array}{l}\text { Total study population } \\
(\mathrm{n}=602)\end{array}$ & $\begin{array}{l}\text { Without GC } \\
(n=216)\end{array}$ & $\begin{array}{l}\text { With GC } \\
(\mathrm{n}=386)\end{array}$ & p Value* \\
\hline Age (years) & 602 & $48 \pm 12$ & $48.9 \pm 11.8$ & $47.5 \pm 12.2$ & 0.210 \\
\hline Female & 602 & $476(79 \%)$ & $176(81.5 \%)$ & $300(77.7 \%)$ & $0.277 \dagger$ \\
\hline $\mathrm{BMI}>30 \mathrm{~kg} / \mathrm{m}^{2}$ & 602 & $83(13.8 \%)$ & $28(13 \%)$ & $55(14.3 \%)$ & $0.643 \dagger$ \\
\hline Diabetes & 602 & $19(3.2 \%)$ & $12(5.6 \%)$ & $7(1.8 \%)$ & $0.012 \dagger$ \\
\hline Smokers & 602 & $291(48.3 \%)$ & $90(41.7 \%)$ & $201(52.1 \%)$ & $0.014 \dagger$ \\
\hline $\begin{array}{l}\text { Hypertension or hypercholesterolaemia or diabetes or } \\
\mathrm{BMI}>30 \mathrm{~kg} / \mathrm{m}^{2} \text { or smoker }\end{array}$ & 602 & $401(66.6 \%)$ & $133(61.6 \%)$ & $268(69.4 \%)$ & $0.050 \dagger$ \\
\hline At least one DMARD treatment from baseline to 7 years & 567 & $541(95 \%)$ & $178(93.2 \%)$ & $363(96.5 \%)$ & $<0.0001 \dagger$ \\
\hline At least one biological agent from baseline to 7 years & 602 & $164(27 \%)$ & $37(17.1 \%)$ & $127(32.9 \%)$ & $<0.0001 \dagger$ \\
\hline $2.6-3.2$ & & $32(5.3 \%)$ & $13(6.02 \%)$ & 19 (4.9\%) & \\
\hline $3.2-5.1$ & & 324 (54\%) & $141(65.3 \%)$ & $183(47.7 \%)$ & \\
\hline$>5.1$ & & $222(37 \%)$ & $49(22.7 \%)$ & $173(45.1 \%)$ & \\
\hline HAQ score & 602 & $1.0 \pm 0.7$ & $0.8 \pm 0.6$ & $1.1 \pm 0.7$ & $<0.0001$ \\
\hline CRP level (mg/L) & 602 & $20.2 \pm 33.9$ & $13.2 \pm 22.1$ & $24.1 \pm 38.5$ & $<0.0001$ \\
\hline CRP level >10 mg/L & 602 & 274 (45.5\%) & 76 (35.2\%) & $198(51.3 \%)$ & $0.0001 \dagger$ \\
\hline $\mathrm{RF}(\mathrm{IU} / \mathrm{mL})$ & 602 & $122.2 \pm 445.7$ & $118.7 \pm 499.6$ & $124.2 \pm 413.1$ & 0.111 \\
\hline IgM-RF positivity & 602 & $317(52.7 \%)$ & $107(49.5 \%)$ & $210(54.4 \%)$ & $0.251 \dagger$ \\
\hline ACPA titres $(\mathrm{IU} / \mathrm{mL})$ & 602 & $555.7 \pm 1577.2$ & $416.6 \pm 1359$ & $633.5 \pm 1683.8$ & 0.0004 \\
\hline ACPA positivity & 602 & 268 (44.5\%) & $76(35.2 \%)$ & $192(49.7 \%)$ & $0.0006 \dagger$ \\
\hline
\end{tabular}

Data are no. (\%) or mean \pm SD.

Bold is related to significant $\mathrm{p}$-Values.

${ }^{*} \mathrm{p}$ Values were assessed by Mann-Whitney $\mathrm{U}$ test.

tp Values were assessed by $\chi^{2}$ test (or Fisher's exact test).

ACPA, anticitrullinated protein antibodies; BMl, body mass index; CRP, C reactive protein; DAS-28-CRP, Disease Activity Score in 28 joints, with C reactive protein level; DMARD,

disease-modifying antirheumatic drug; ESPOIR, Etude et Suivi des POlyarthrites Indifférenciées Récentes; GC, glucocorticoids; HAQ, Health Assessment Questionnaire; NSAIDs,

non-steroidal anti-inflammatory drugs; RF, rheumatoid factor; mSHS, van der Heijde-modified Sharp score.

$>3.2)$ experienced significantly more events than those without GC $(\mathrm{p}=0.006)$. On weighted Cox proportional-hazards analysis (IPTW) (see online supplementary figure S1) including age, gender, history of hypertension and GC treatment, the composite outcome did not differ with and without GC $(\mathrm{p}=0.520$; $\mathrm{HR}=0.889 ; 95 \%$ CI 0.620 to 1.273 ). The covariates with significant effect on the composite outcome were age $(p=0.02$; $\mathrm{HR}=1.636 ; 95 \%$ CI 1.085 to 2.467$)$ and gender $(\mathrm{p}=0.003$; $\mathrm{HR}=1.809 ; 95 \%$ CI 1.224 to 2.674 ).

In the analysis of the 657 patients including those with a history, the Cox IPTW analysis (see online supplementary figure S2) included age, gender, history of hypertension, history of CVD or factures or severe infections, RF, CRP level, BMI $>30 \mathrm{~kg} / \mathrm{m}^{2}$ and GC treatment. Again, the composite outcome did not differ with and without GC $(p=0.767, H R=0.951$; 95\% CI 0.684 to 1.323 ).

Finally, regarding the four-level categorical variable representing cumulative dose and duration of GC treatment, neither the cumulative dose nor duration of GC treatment had an effect on survival (log-rank test, $\mathrm{p}=0.79$ and $\mathrm{p}=0.57$ ). Also, with two stratified Cox models used to assess the association between the covariates age, gender and history of hypertension and the composite outcome, while controlling for the four-level categorical variable, the results were similar to those with the initial Cox model (see online supplementary figure S3 and table S2).

\section{DISCUSSION}

In a cohort of very early RA from a real-life setting monitored for 7 years, we investigated the association between exposure to GC treatment and classical major safety events related to GC (death, CVD, severe infection, fracture). This 7-year data analysis of the ESPOIR cohort did not show any significant difference between patients with RA with and without GC treatment in terms of major safety events. Most of the patients who received GC therapy started GC during the first 6 months and received low-dose therapy. These results support the good safety profile of low-dose GC therapy for early RA and agree with the recent work by Strehl et al. ${ }^{20}$

GC are considered a bridging therapy, with short-term symptomatic $^{2122}$ and structural effects. However, the GC risk/benefit balance has little evidence base, with most recent data provided by observational studies. These studies provide the opportunity to explore the real-life tolerability profile of GC, with doses and duration commonly used in daily practice, but often present bias 
Table 2 Primary outcome at 7 years (death or cardiovascular disease or severe infection or fracture) in the total sample and patients with and without GC

\begin{tabular}{|c|c|c|c|c|}
\hline & $\begin{array}{l}\text { Total study } \\
\text { population } \\
(\mathrm{n}=602)\end{array}$ & $\begin{array}{l}\text { Without GC } \\
(n=216)\end{array}$ & $\begin{array}{l}\text { With GC } \\
(n=386)\end{array}$ & p Value* \\
\hline Primary outcome & $65(10.8 \%)$ & $21(9.7 \%)$ & $44(11.4 \%)$ & 0.520 \\
\hline Death & 7 & 1 & 6 & 0.430 \\
\hline Cardiovascular disease & 14 & 3 & 11 & 0.400 \\
\hline $\begin{array}{l}\text { Coronary artery } \\
\text { disease }\end{array}$ & 8 & 2 & 6 & - \\
\hline Stroke & 5 & 1 & 4 & - \\
\hline Heart failure & 1 & 0 & 1 & - \\
\hline Severe infections & 19 & 3 & 16 & 0.090 \\
\hline Pneumonia & 4 & 2 & 2 & - \\
\hline Urinary tract infection & 7 & 0 & 7 & - \\
\hline Digestive & 3 & 1 & 2 & - \\
\hline Cutaneous & 3 & 0 & 3 & - \\
\hline Other & 2 & 0 & 2 & - \\
\hline Fractures & 25 & 14 & 11 & 0.150 \\
\hline
\end{tabular}

such as confounding by indication. ${ }^{20}$ As well, the evidence from randomised clinical trials is scarce. ${ }^{11} 23$

The GC tolerability profile has been reported to depend both on the duration of exposure and dose. ${ }^{5}$ Indeed, in addition to a better tolerability profile of a low-dose than high-dose regimen, ${ }^{24}{ }^{25}$ long-term use of low-dose GC has been associated with increased mortality as compared with shorter exposures. ${ }^{26}$ Most notably, two recent studies suggested a dose-dependent increase in mortality in $\mathrm{RA}^{27}{ }^{28}$; del Rincón et al ${ }^{27}$ revealed a daily threshold dose of $8 \mathrm{mg}$ at which all-cause mortality increased with GC dose (adjusted HR=1.78; 95\% CI 1.22 to 2.60), and in the German register Rheumatoid Arthritis oBservation of BIologic Therapy (RABBIT), use of GC $>5 \mathrm{mg} /$ day was associated with increased mortality risk, independent of RA activity. ${ }^{28}$ Moreover, a 10-year follow-up study examined cardiovascular events and deaths in early patients with RA with no history of CVD who were included in a recent open-label randomised trial of low-dose prednisolone $(7.5 \mathrm{mg} /$ day $)$ over the first 2 years of early RA Better Anti-Rheumatic PharmacOTherapy (BARFOT+): low-dose prednisolone use was associated with increased incidence of cerebrovascular events and, although not significant, increased mortality. ${ }^{29}$ Long-term follow-up of Computer Assisted Management in Early Rheumatoid Arthritis (CAMERA II) patients with early RA who received prednisone at $10 \mathrm{mg} /$ day for at least 2 years revealed increased cardiovascular risk and, although not significant, increased mortality. ${ }^{30}$

In our study, most of the patients who took GC received low-dose GC, $<5 \mathrm{mg}$ /day (mean dosage during the entire follow-up $3.1 \pm 2.9 \mathrm{mg} /$ day), for which the literature supports an acceptable safety profile. ${ }^{31}$

Cardiovascular tolerance of GC remains controversial. In one meta-analysis of observational studies, GC usage was associated with increased risk of all cardiovascular events (relative risk $=1.47$; $95 \%$ CI 1.34 to 1.60 ), including myocardial infarction, heart failure and stroke. ${ }^{32}$ In another systematic literature review, low-dose GC ( $<10 \mathrm{mg} /$ day) was associated with major cardiovascular events in four of six studies. ${ }^{33}$
GC therapy has been associated with increased risk of severe infections. ${ }^{34-36}$ One systematic review noted the paucity of data on the association between low-dose GC $(<10 \mathrm{mg} /$ day prednisone) and risk of infection. ${ }^{37}$ In one recent study evaluating patients with RA aged $>65$ years, the risk of serious infection was increased $30 \%, 46 \%$ or $100 \%$ with $5 \mathrm{mg}$ prednisolone used continuously for the last 3 or 6 months or 3 years, respectively, as compared with no use. ${ }^{38}$ The increased risk of severe infection was also proportional to the cumulative dosage over 2-3 years.

Potential limitations of the present study are those inherent to observational studies, with potential confounders that could not be taken into account. Moreover, as in many cohort studies, the data are mostly declarative. The events and comorbid diseases were reported by patients, and a potential recall bias cannot be excluded. HAQ and disease activity variables that were selected by the logistic regression analysis and included in the PS were baseline variables, which could also be considered a limitation, because the relationship between GC-related events and disease activity evolution over time could not be evaluated. Using a composite end point is controversial, mostly because it may emphasise each patient's first outcome. Nevertheless, GC toxicity is multifaceted and this way of assessing the most important adverse events may help identify the net effect of GC. We also decided to use a composite outcome including the four most relevant adverse events related to GC treatment, mainly to increase the number of events and to cover the four most worrisome adverse effects of GC therapy defined by a panel of rheumatologists. ${ }^{39}$ Moreover, we could not perform a dose-response analysis because of the low doses the patients received. Finally, the relatively small number of incident events might have implied relatively low power.

The present study has many strengths. First, the ESPOIR cohort offered a unique opportunity to explore the long-term impact of GC in very early RA, in a real-life scenario. Importantly, all participants who received GC started treatment after entering the cohort, and only incident safety events were considered. To our knowledge, this is the first cohort study specifically designed to assess (among other data) GC adverse effects and to report the long-term tolerability profile of GC use in early RA. Second, the present 7-year duration of follow-up provides a sufficient period of observation to ensure a true association between GC therapy and adverse effects related to long-term treatment. Third, the ESPOIR cohort has inherent qualities, including the prospective independent collection of data and low rate of missing data and dropout. ${ }^{12}$

Finally, using a PS in this study limited the indication bias, thus reinforcing the association between GC and long-term adverse events. Indeed, several factors such as RA disease activity and comorbidities or history may have an impact on GC prescription. For instance, in one recent observational study, the association between $\mathrm{GC}$ use and increased incidence of CVD was negated after adjustment for disease activity and severity, which suggests an effect confounded by indication due to high disease activity. ${ }^{40}$ The propensity for prescribing GC is important to consider when evaluating the association between GC use and related outcomes, given that patients with RA with more active disease might have more likely received GC, and conversely, those with history of CVD or severe infections or fractures might have less frequently received GC. Therefore, using a PS allowed for adjusting of patterns that are difficult to fully account for by adjusting for only general and RA-related characteristics in regression modelling. In the ESPOIR cohort, several characteristics differed between patients with and without GC, which highlights the value of using the PS in evaluating the relationship between GC use and outcomes. 


\section{CONCLUSIONS}

This 7-year data analysis of the ESPOIR cohort did not show any significant difference in major safety events among patients with RA with and without GC treatment. These data support the good safety profile of very low-dose GC therapy in early RA. Although our findings need further confirmation, they strongly support the current recommendations ${ }^{3}$ that GC should be used for early RA, with DMARDs, for the shortest period and at the lowest possible dose.

Acknowledgements The authors thank Mohamed Belkacemi for statistical advice.

Contributors All authors participated in the conception and design of the study, contributed to the acquisition of data, participated in the analysis and interpretation of data and read, revised and approved the final manuscript. NR and JPD performed and are responsible for the statistical analyses.

Funding CR received a grant (2838) from the French Society of Rheumatology (SFR). The Etude et Suivi des POlyarthrites Indifférenciées Récentes (ESPOIR) cohort was supported by an unrestricted grant from Merck Sharp and Dohme for the first 5 years. Part of the biological database was supported by two additional grants from Institut National de la Santé et de la Recherche Médicale (INSERM), and the ESPOIR cohort study was also supported by the French Society of Rheumatology, AbbVie, Pfizer and Roche Chugai.

Competing interests None declared.

Ethics approval EC Montpellier University Hospital, France.

Provenance and peer review Not commissioned; externally peer reviewed.

\section{REFERENCES}

1 Kirwan JR, Bijlsma JW, Boers $M$, et al. Effects of glucocorticoids on radiological progression in rheumatoid arthritis. Cochrane Database Syst Rev 2007;(1) CD006356

2 Kavanaugh A, Wells AF. Benefits and risks of low-dose glucocorticoid treatment in the patient with rheumatoid arthritis. Rheumatology (Oxford) 2014;53:1742-51.

3 Smolen JS, Landewé $\mathrm{R}$, Breedveld FC, et al. EULAR recommendations for the management of rheumatoid arthritis with synthetic and biological disease-modifying antirheumatic drugs: 2013 update. Ann Rheum Dis 2014;73:492-509.

4 Singh JA, Saag KG, Bridges SL Jr, et al. 2015 American College of Rheumatology Guideline for the Treatment of Rheumatoid Arthritis. Ann Rheum Dis 2016:68:1-26

5 Ethgen 0, de Lemos Esteves F, Bruyere 0, et al. What do we know about the safety of corticosteroids in rheumatoid arthritis? Curr Med Res Opin 2013:29:1147-60.

6 Black RJ, Joseph RM, Brown B, et al. Half of U.K. patients with rheumatoid arthritis are prescribed oral glucocorticoid therapy in primary care: a retrospective drug utilisation study. Arthritis Res Ther 2015;17:375.

7 Albrecht K, Callhoff J, Schneider M, et al. High variability in glucocorticoid starting doses in patients with rheumatoid arthritis: observational data from an early arthritis cohort. Rheumatol Int 2015;35:1377-84.

8 McKeown E, Bykerk VP, De Leon F, et al. Quality assurance study of the use of preventative therapies in glucocorticoid-induced osteoporosis in early inflammatory arthritis: results from the CATCH cohort. Rheumatology (Oxford) 2012;51:1662-9.

9 Cardiel MH, Pons-Estel BA, Sacnun MP, et al. Treatment of early rheumatoid arthritis in a multinational inception cohort of Latin American patients: the GLADAR experience. J Clin Rheumatol 2012;18:327-35.

10 Gaujoux-Viala C, Gossec L. When and for how long should glucocorticoids be used in rheumatoid arthritis? International guidelines and recommendations. Ann $\mathrm{N}$ Y Acad Sci 2014;1318:32-40.

11 Santiago T, da Silva JA. Safety of glucocorticoids in rheumatoid arthritis: evidence from recent clinical trials. Neuroimmunomodulation 2015:22:57-65.

12 Combe B, Rincheval N. Early lessons from the recent-onset rheumatoid arthritis cohort ESPOIR. Joint Bone Spine 2015;82:13-17

13 Combe B, Benessiano J, Berenbaum F, et al. The ESPOIR cohort: a ten-year follow-up of early arthritis in France: methodology and baseline characteristics of the 813 included patients. Joint Bone Spine 2007:74:440-5.

14 Combe B, Rincheval N, Benessiano J, et al. Five-year favorable outcome of patients with early rheumatoid arthritis in the 2000s: data from the ESPOIR cohort. J Rheumatol 2013;40:1650-7

15 Aletaha D, Neogi T, Silman AJ, III, et al. 2010 rheumatoid arthritis classification criteria: an American College of Rheumatology/European League Against Rheumatism collaborative initiative. Ann Rheum Dis 2010;69:1580-8.

16 Aviña-Zubieta JA, Abrahamowicz M, De Vera MA, et al. Immediate and past cumulative effects of oral glucocorticoids on the risk of acute myocardial infarction in rheumatoid arthritis: a population-based study. Rheumatology (Oxford) 2013;52:68-75.
17 Buttgereit F, da Silva JA, Boers M, et al. Standardised nomenclature for glucocorticoid dosages and glucocorticoid treatment regimens: current questions and tentative answers in rheumatology. Ann Rheum Dis 2002;61:718-22.

18 Robins JM, Hernán MA, Brumback B. Marginal structural models and causal inference in epidemiology. Epidemiology 2000;11:550-60.

19 Brookhart MA, Schneeweiss S, Rothman KJ, et al. Variable selection for propensity score models. Am J Epidemiol 2006;163:1149-56.

20 Strehl C, Biilsma JW, de Wit M, et al. Defining conditions where long-term glucocorticoid treatment has an acceptably low level of harm to facilitate implementation of existing recommendations: viewpoints from an EULAR task force. Ann Rheum Dis 2016:75:952-7.

21 Alten R, Grahn A, Holt RJ, et al. Delayed-release prednisone improves fatigue and health-related quality of life: findings from the CAPRA-2 double-blind randomised study in rheumatoid arthritis. RMD Open 2015;1:e000134.

22 Cutolo M. Glucocorticoids and chronotherapy in rheumatoid arthritis. RMD Open 2016;2:e000203.

23 Bakker MF, Jacobs JW, Welsing PM, et al. Low-dose prednisone inclusion in a methotrexate-based, tight control strategy for early rheumatoid arthritis: a randomized trial. Ann Intern Med 2012;156:329-39.

24 Da Silva JA, Jacobs JW, Kirwan JR, et al. Safety of low dose glucocorticoid treatment in rheumatoid arthritis: published evidence and prospective trial data. Ann Rheum Dis 2006:65:285-93.

25 Hoes JN, Jacobs JW, Verstappen SM, et al. Adverse events of low- to medium-dose oral glucocorticoids in inflammatory diseases: a meta-analysis. Ann Rheum Dis 2009;68:1833-8

26 Sihvonen S, Korpela M, Mustonen J, et al. Mortality in patients with rheumatoid arthritis treated with low-dose oral glucocorticoids. A population-based cohort study. J Rheumatol 2006:33:1740-6.

27 del Rincón I, Battafarano DF, Restrepo JF, et al. Glucocorticoid dose thresholds associated with all-cause and cardiovascular mortality in rheumatoid arthritis. Arthritis Rheum 2014:66:264-72.

28 Listing J, Kekow J, Manger B, et al. Mortality in rheumatoid arthritis: the impact of disease activity, treatment with glucocorticoids, TNF $\alpha$ inhibitors and rituximab. Ann Rheum Dis 2015;74:415-21.

29 Ajeganova S, Svensson B, Hafström I, BARFOT Study Group. Low-dose prednisolone treatment of early rheumatoid arthritis and late cardiovascular outcome and survival: 10-year follow-up of a 2-year randomised trial. BMJ Open 2014;4:e004259.

30 de Hair $\mathrm{M}$, IJff $\mathrm{N}$, Jacobs J, et al. Long-term adverse events after daily concomitant treatment with $10 \mathrm{mg}$ prednisone in the 2-year computer assisted management in early rheumatoid arthritis trial-II [abstract]. Arthritis Rheumatol 2015;67(Suppl 10):856-7

31 Caporali R, Todoerti M, Scirè CA, et al. Oral low-dose glucocorticoids should be included in any recommendation for the use of non-biologic and biologic disease-modifying antirheumatic drugs in the treatment of rheumatoid arthritis. Neuroimmunomodulation 2015;22:104-11.

32 Roubille $C$, Richer $V$, Starnino $T$, et al. The effects of tumour necrosis factor inhibitors, methotrexate, non-steroidal anti-inflammatory drugs and corticosteroids on cardiovascular events in rheumatoid arthritis, psoriasis and psoriatic arthritis: a systematic review and meta-analysis. Ann Rheum Dis 2015;74:480-9.

33 Ruyssen-Witrand A, Fautrel B, Saraux A, et al. Cardiovascular risk induced by low-dose corticosteroids in rheumatoid arthritis: a systematic literature review. Joint Bone Spine 2011;78:23-30.

34 Wolfe F, Caplan L, Michaud K. Treatment for rheumatoid arthritis and the risk of hospitalization for pneumonia: associations with prednisone, disease-modifying antirheumatic drugs, and anti-tumor necrosis factor therapy. Arthritis Rheum 2006;54:628-34.

35 Lacaille D, Guh DP, Abrahamowicz M, et al. Use of nonbiologic disease-modifying antirheumatic drugs and risk of infection in patients with rheumatoid arthritis Arthritis Rheum 2008:59:1074-81.

36 Haraoui B, Jovaisas A, Bensen WG, et al. Use of corticosteroids in patients with rheumatoid arthritis treated with infliximab: treatment implications based on a real-world Canadian population. RMD Open 2015;1:e000078.

37 Ruyssen-Witrand A, Fautrel B, Saraux A, et al. Infections induced by low-dose corticosteroids in rheumatoid arthritis: a systematic literature review. Joint Bone Spine 2010;77:246-51.

38 Dixon WG, Abrahamowicz M, Beauchamp ME, et al. Immediate and delayed impact of oral glucocorticoid therapy on risk of serious infection in older patients with rheumatoid arthritis: a nested case-control analysis. Ann Rheum Dis 2012;71:1128-33.

39 van der Goes MC, Jacobs JW, Boers M, et al. Patient and rheumatologist perspectives on glucocorticoids: an exercise to improve the implementation of the European League Against Rheumatism (EULAR) recommendations on the management of systemic glucocorticoid therapy in rheumatic diseases. Ann Rheum Dis 2010;69:1015-21.

40 van Sijl AM, Boers $M$, Voskuyl $A E$, et al. Confounding by indication probably distorts the relationship between steroid use and cardiovascular disease in rheumatoid arthritis: results from a prospective cohort study. PLOS ONE 2014;9: e87965. 